\title{
Representing Subgroups of Finitely Presented Groups by Quotient Subgroups
}

\author{
Alexander Hulpke
}

\section{CONTENTS}

1. Creation of Quotient Representations and Easy Calculations

2. Subdirect Products

3. Iterated Quotient Representations

4. Polycyclic-by-Finite Groups

5. An Example

Acknowledgements

References
This article proposes to represent subgroups of finitely presented groups by their image in a quotient. It gives algorithms for basic operations in this representation and investigates how iteration of this approach can be used to extend known quotients groups with a solvable normal subgroup.

Traditionally, algorithms in group theory have represented a subgroup $U$ of a finitely presented group $G$ either by a set of generators (as words in the generators of the full group) or via a coset table.

Both representations have disadvantages. A coset table for $U$ has $[G: U]$ rows, so the space requirement for storage can be large, which in practice restricts this representation to index a few million at best. The other method of representation, via a generating set, is not particularly suited to calculations: many algorithms compute a coset table as a first step and effectively work with it, discarding the generating set. Furthermore, for subgroups obtained as output of other algorithms, generating sets often are not what a user wants. The standard way to obtain generators is as Schreier generators; since this is a process that "lives" in a free group, the number of generators produced grows linearly with the index, and any reduction of this number (if possible at all) would require further work.

The first aim of this article is to introduce a different representation, namely as a subgroup of a quotient: Given a group $G$ and a homomorphism $\varphi$, we represent a subgroup $U$ which is a full preimage under $\varphi$ by the pair $(\varphi, U \varphi)$. We call $U$ a quotient subgroup and the pair $(\varphi, U \varphi)$ the quotient representation of $U$.

Such representation comes naturally to systems such as Magnus [Magnus 1997], quotpic [Holt and Rees 1993] or XGAP [Celler and Neunhöffer 1999] 
having a graphical user interface in which subgroups are objects whose generators or coset table are (usually) never displayed.

We will show how such representations can be created, and how to calculate with subgroups in this representation. We will then examine how this approach can be iterated in an attempt to extend known quotients.

The approach has been implemented by the author in GAP [GAP 2000] and is used there as a default method for many operations for subgroups of finitely presented groups.

\section{CREATION OF QUOTIENT REPRESENTATIONS AND EASY CALCULATIONS}

If $U \leq G$ is of finite index a Todd-Coxeter coset enumeration [Todd and Coxeter 1936] will yield a permutation representation $\varphi$ of $G$ on the cosets of $U$. The image of $U$ is the stabilizer $\operatorname{Stab}_{G \varphi}(1)$. So the quotient representation of $U$ is $\left(\varphi, \operatorname{Stab}_{G \varphi}(1)\right)$.

Similarly, the low-index subgroup algorithm [Sims 1994, 5.6] can be considered to produce admissible homomorphisms $\varphi$ into permutation groups, the quotient representation of the subgroups found is again of the form $\left(\varphi, \operatorname{Stab}_{G \varphi}(1)\right)$. A possible variant is the "G-quotient" algorithm [Holt and Rees 1992; Hulpke 1996, V.5], which, using a similar search, finds quotients isomorphic to a given group.

Quotient algorithms such as the $p$-quotient [Macdonald 1974], the nilpotent quotient [Wamsley 1974; Nickel 1996], the solvable quotient [Plesken 1987; Niemeyer 1994; Brückner 1998], and the polycyclic quotient [Lo 1998] all return a homomorphism $\varphi$. Subgroups induced by this quotient are naturally given in quotient representation.

If $\psi$ is a homomorphism with domain $G$, the quotient representation of $\operatorname{ker} \psi$ is $(\psi,\langle\cdot\rangle)$. In particular, this allows us to represent the intersections of the conjugates of a subgroup (whose index can be $n$ !, making coset tables very space-consuming) in a quotient representation using the same homomorphism $\varphi$.

This provides a natural way to represent commutator subgroups: $G^{\prime}$ is the kernel of the epimorphism on the largest abelian quotient (whose structure we obtain by abelianizing the presentation). If $N \triangleleft G$, the commutator subgroup $[G, N]$ has a quotient in
$G$ we obtain by adding the commutators $[g, n]$ (for $g$ and $n$ running through a generating set of $G$ and $N$, respective) to the relators of $G$. A lower central series is obtained by taking for $\varphi$ the epimorphism of $G$ onto its largest nilpotent quotient (as found by a Nilpotent Quotient Algorithm) $Q$, the terms of the lower central series of $G$ are the preimages under $\varphi$ of the lower central series of $Q$.

For a more complex example consider $\mathrm{PSL}_{2}(\mathbb{Z})$, which is isomorphic to a free product $C_{2} * C_{3}$. A congruence subgroup such as

$$
\Gamma_{0}(N)=\left\{\left(\begin{array}{ll}
a & b \\
c & d
\end{array}\right) \mid c \equiv 0(\bmod N)\right\}
$$

is the preimage of the group of upper triangular matrices under the "reduction-mod- $N$ " homomorphism. So we can represent these subgroups (and calculate with them) in quotient representation as subgroups of $C_{2} * C_{3}$.

If a subgroup $U \leq G$ is given in quotient representation $(\varphi, U \varphi)$, we can easily obtain the coset table representation and the representation by subgroup generators, as we shall see forthwith. Thus the quotient representation will still allow the use of all existing algorithms.

A coset table for $U$ in $G$ represents permutations for the action of $G$ on the cosets of $U$. This permutation action has the same image as the action of $G \varphi$ on the cosets of $U \varphi$, and we can compute permutations for this action easily if a membership test for $U \varphi$ is available.

To compute generators of $U$, we can then simply take this permutation action on the cosets, and compute Schreier generators for it. But in almost all cases subgroup generators will not be required for further calculations - they would just be in the way and take up space.

For a membership test, observe that $x \in U$ if and only if $x \varphi \in U \varphi$, since $\operatorname{ker} \varphi \leq U$.

\section{SUBDIRECT PRODUCTS}

Now assume that $U, V \leq G$ are subgroups given by the quotient representations $(\varphi, U \varphi)$ and $(\psi, V \psi)$. We set $A:=G \varphi$ and $B:=G \psi$ and define a homomorphism $\varepsilon: G \rightarrow A \times B, g \mapsto(g \varphi, g \psi)$. Its kernel is the intersection $(\operatorname{ker} \varphi \cap \operatorname{ker} \psi$ ). The image $G \varepsilon$ is a subdirect product of $A$ and $B$ [Remak 1930]. 
Denote the normal subgroup generated by both kernels by $M=\langle\operatorname{ker} \varphi, \operatorname{ker} \psi\rangle$. Then $M$ is mapped to two normal subgroups $E:=M \varphi \triangleleft A$ and $F:=$ $M \psi \triangleleft B$. By the homomorphism theorem we have $A / E \cong G / M \cong B / F$. The structure is this:

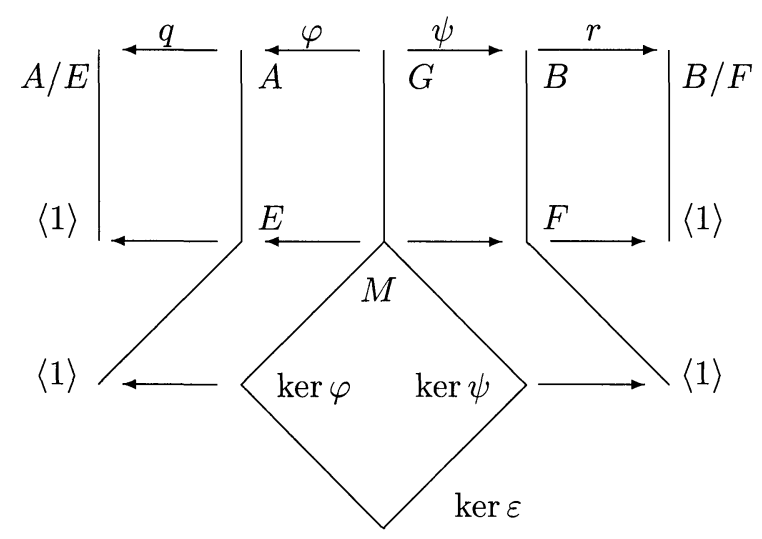

If $A$ and $B$ are both represented as permutation groups, the direct product $A \times B$ can be represented again as a permutation group (on the disjoint union of the domains of $A$ and $B$ ) and we can use this representation to compute in its subgroup $G \varepsilon$. Similarly, if $A$ and $B$ are both matrix groups over the same field, $A \times B$ can be represented as matrix group again by block matrices. If $A$ and $B$ are given by polycyclic presentations, we can easily write down a polycyclic presentation of $A \times B$.

We now map a generating system $\underline{\boldsymbol{g}}=\left(g_{1}, \ldots, g_{l}\right)$ under both projections and get generating systems $\underline{\boldsymbol{a}}:=\underline{\boldsymbol{g}} \varphi$ of $A$ and $\underline{\boldsymbol{b}}:=\underline{\boldsymbol{g}} \psi$ of $B$. Let $\langle\underline{\boldsymbol{x}} \mid \mathcal{R}\rangle$ be a presentation for $B$ on generators $\underline{\boldsymbol{x}}$ corresponding to $\underline{\boldsymbol{b}}$. Then the epimorphism from the free group $X=\langle x\rangle$ defined by $\beta:=(X \rightarrow B, \underline{\boldsymbol{x}} \mapsto \underline{\boldsymbol{b}})$ factors via $\gamma:=(X \rightarrow G, \underline{\boldsymbol{x}} \mapsto \underline{\boldsymbol{g}})$ as $\beta=\gamma \psi$. The kernel ker $\psi$ thus is the image of $\operatorname{ker} \beta$ under $\gamma$, and therefore $E=(\operatorname{ker} \beta) \gamma \varphi$.

For a subset $\mathcal{S} \subset G$ of a group $G$, we denote by $\langle\mathcal{S}\rangle_{\mathrm{NT}}$ the normal closure of $\mathcal{S}$, that is the smallest normal subgroup of $G$ containing all elements of $\mathcal{S}$.

By the definition of a presentation $\operatorname{ker} \beta=\langle\mathcal{R}\rangle_{\mathrm{NT}}$ Therefore $E$ is the normal closure in $A$ of the set $\{r(\underline{a}) \mid r \in \mathcal{R}\}$ of relators of $B$ evaluated in $\underline{\boldsymbol{a}}$. We call this subgroup the cokernel of the relation $\zeta: B \rightarrow A$ obtained by extending the mapping $(\underline{\boldsymbol{b}} \mapsto$ a) homomorphically.

To compute $E$, it is sufficient to have a procedure to evaluate a set of defining relators in given gener- ators, there is no need to actually write down a full presentation. Such a procedure is already available as part of the functionality for computing kernels of homomorphisms [Leedham-Green et al. 1991].

If $\underline{\boldsymbol{v}} \subset B$ is a generating set of $V \psi$, then $V$ is generated by ker $\psi$ together with representatives of preimages of $\underline{\boldsymbol{v}}$ under $\psi$. Therefore $V \varphi$ is the subgroup of $A$ generated by $E$ and representatives of images of $\underline{\boldsymbol{v}}$ under $\zeta$.

Similarly $F$ is the cokernel of the homomorphic closure $\xi$ of $(\underline{\boldsymbol{a}} \mapsto \underline{\boldsymbol{b}})$ and $U \psi=\langle F, u \xi \mid u \in \underline{\boldsymbol{u}}\rangle$ for a generating set $\underline{u}$ of $U \varphi$.

We can therefore compute $E, F$, and the converse images $U \psi$ and $V \varphi$ directly from the constituents $A$ and $B$ with the generating set $\underline{a}$ and $\underline{b}$. Furthermore, note that $U \varepsilon \leq A \times B$ is the full preimage of $U \psi$ under the projection form $G \varepsilon$ to $A$.

This gives us all the necessary input for the following computations:

Lemma 2.1. (a) $(\varepsilon, U \varepsilon \cap V \varepsilon)$ is a quotient representation of $U \cap V$.

(b) $(\varphi,\langle U \varphi, V \varphi\rangle)$ is a quotient representation of $\langle U, V\rangle$.

(c) $\left(\varepsilon, N_{U \varepsilon}(V \varepsilon)\right)$ is a quotient representation of $N_{U}(V)$.

(d) $\left(\varphi, \bigcap_{g \in G \varphi}(U \varphi)^{g}\right)$ is a quotient representation of $\bigcap_{g \in G} U^{g}$.

(e) Representatives for the double cosets $U \backslash G / V$ are given by (the preimages under $\varphi$ of) representatives for the double cosets $U \varphi \backslash G \varphi / V \varphi$.

Proof. Since $\operatorname{ker} \varepsilon \leq U, V$, part (a) follows immediately. Similarly (b) follows from $\operatorname{ker} \varphi \leq U \leq\langle U, V\rangle$. For (c) observe that $\operatorname{ker} \varepsilon \leq V \leq N_{G}(V)$; d) follows as $\operatorname{ker} \varphi \leq \bigcap_{g \in G} U^{g}$. Finally in e), the double cosets are orbits of $V$ on the cosets of $U$, for which $\varphi$ is a bijection.

To test containment, we do not need to form the subdirect product:

Lemma 2.2. $V \leq U$ if and only if $E \leq U \varphi$ and $V \psi \leq$ $U \psi$.

Proof. Since ker $\psi \leq V$ and since subset relations are preserved by homomorphisms, the conditions are certainly necessary.

Conversely, $E \leq U \varphi$ implies that $\operatorname{ker} \psi \leq U$. In this situation it is sufficient to test containment in 
the image of $\psi$, which proves sufficiency of the criterion.

Inclusion in both directions gives an equality test.

\section{A. Presentations for Subdirect Products}

In the course of this article we shall need a presentation for a subdirect product. The following approach must be well-known; it is only given here because I've not been able to find explicit literature references.

We consider a subdirect product of two finitely generated groups $A$ and $B$ via an isomorphism of their factor groups $A / E$ with $B / F$ and let

$$
\varphi: A \curlywedge B \rightarrow A, \quad \psi: A \curlywedge B \rightarrow B
$$

be the component projections. If $X$ is a generating set for $A \curlywedge B, \hat{X}=X \varphi$ and $\tilde{X}=X \psi$ are generating sets for $A$ and for $B$. We assume we are given presentations for the constituent groups $A \cong\langle\hat{X} \mid \mathcal{R}\rangle$ and $B \cong\langle\hat{X} \mid \mathcal{S}\rangle$ in these generators (script letters will be used to denote sets of relators). We shall write $w(X)$ do denote an abstract word in $X$.

Then $\operatorname{ker} \varphi=\langle r(X) \mid r \in \mathcal{R}\rangle_{\mathrm{NT}}$. By adding conjugates, we extend this set to $Y_{2}=\left\{r(X)^{a} \mid r \in \mathcal{R}\right.$ for some $a \in\langle X\rangle\}$ such that $F=(\operatorname{ker} \varphi) \psi=\left\langle Y_{2} \psi\right\rangle$. We denote by $Y_{B}=Y_{2} \psi$ the images of $Y_{2}$ in $B$. Given the presentations for $A$ and $B$ we can verify that enough conjugates are taken by checking that $\left[B:\left\langle Y_{B}\right\rangle\right]=[A: E]$. Rewriting the presentation in these subgroup generators we furthermore get a presentation $\left\langle Y_{2} \mid \mathcal{R}_{F}\right\rangle$ for $F$. Finally, using rewriting in $\left\langle Y_{B}\right\rangle \leq B$, we obtain conjugation relations $\mathcal{R}_{C}=\left\{y^{x}=w\left(Y_{2}\right) \mid y \in Y_{2}, x \in \tilde{X}\right\}$.

Lemma 2.3. $A$ presentation for $A \curlywedge B$ is given by

$$
\begin{aligned}
&\left\langle X, Y_{2}\right| y=w(X), \\
& r\left(Y_{2}\right),
\end{aligned}
$$

where $y$ ranges over $Y_{2}, r$ ranges over $\mathcal{R}_{F}$, and the relations (2-1) are the definitions of $Y_{2}$ as words in $X$.

Proof. The relations given certainly hold in $A \curlywedge B$, since they were obtained from $A \curlywedge B$.

If we add relations $Y_{2}=1$ to the given presentation, the relations of type $(2-1)$ become conjugates
$r(X)^{x}$ (with $r \in \mathcal{R}, a \in\langle X\rangle$ ) containing the whole of $\mathcal{R}$. The resulting group is thus generated by $X$ and isomorphic to $A$.

The kernel of this projection onto $A$ is generated by conjugates of $Y_{2}$, and relations of type (2-3) show that $\left\langle Y_{2}\right\rangle$ is conjugation invariant. Furthermore the relations (2-2) show that $\left\langle Y_{2}\right\rangle$ must be isomorphic to a factor group of $F$.

Using Tietze transformations and the expressions $(2-1)$ of $Y_{2}$ as words in $X$, we can get rid of the auxiliary generators $Y_{2}$ and obtain a presentation in terms of $X$.

Corollary 2.4. If $F$ is a free group, $\mathcal{R}, \mathcal{S}$ are finite sets generating normal subgroups $\langle\mathcal{R}\rangle_{\mathrm{NT}},\langle\mathcal{S}\rangle_{\mathrm{NT}} \triangleleft F$ and $\left[F:\langle\mathcal{R}, \mathcal{S}\rangle_{\mathrm{NT}}\right]$ is finite, the intersection $\langle\mathcal{R}\rangle_{\mathrm{NT}} \cap$ $\langle\mathcal{S}\rangle_{\mathrm{NT}} \triangleleft F$ is finitely generated as a normal subgroup.

\section{ITERATED QUOTIENT REPRESENTATIONS}

As mentioned above, if $U<G$ is given in quotient representation $(\varphi, U \varphi)$, we can obtain a coset table for $U$ in $G$ from the action of $G \varphi$ on the cosets of $U \varphi$.

Using Reidemeister rewriting [Magnus et al. 1966, 2.3 ], it is then possible to compute from this coset table a set of generators of $U$ and a presentation in these generators [Havas 1974; Neubüser 1982]. (In practice, one would simplify the resulting presentation using Tietze transformations before working with it.)

This new presentation (and the rewriting process for subgroup elements) thus enables us to evaluate homomorphisms with domain $U$. One can then again apply algorithms to $U$, obtaining subgroups of $U$ in quotient representation.

If $V \leq U$ is such a subgroup, given by the quotient representation $(\alpha, V \alpha)$ with $\alpha$ defined on $U$, we want to get a quotient representation for $V$ as a subgroup of $G$. We can achieve this using the embedding theorem for wreath products [Krasner and Kaloujnine 1951]:

Let $\beta$ be the permutation action of $G$ on the cosets of $U$ and set $C=U \alpha$ and $D=G \beta$. We label the cosets of $U$ in $G$ from 1 to $n=[G: U]$ and pick coset representatives $\left\{r_{i}\right\}$ such that $r_{i} \beta$ maps 1 to $i$. Now 
we define a mapping $\gamma=\alpha \uparrow_{(\beta)}^{G}: G \rightarrow C \imath D=C^{\times n} . D$ by:

$$
\begin{aligned}
& \gamma: g \mapsto\left(\left(r_{1} \cdot g \cdot r_{\left(1^{g}\right)}^{-1}\right) \alpha, \ldots,\left(r_{i} \cdot g \cdot r_{\left(i^{g}\right)}^{-1}\right) \alpha, \ldots,\right. \\
&\left.\left(r_{n} \cdot g \cdot r_{\left(n^{g}\right)}^{-1}\right) \alpha, g^{\beta}\right) \in C^{\times n} \cdot D
\end{aligned}
$$

(writing $i^{x}$ for $i^{(x \beta)}$ and noting that $r_{i} x r_{\left(i^{x}\right)}^{-1} \in V$ ).

Remark 3.1. If the image of $\alpha$ is a permutation group, $C$ < $D$ can be represented as a permutation group again. If $\alpha$ is a matrix representation, $\gamma$ can be considered as the induced representation $\alpha \uparrow_{(\beta)}^{G}$. (This proves that $\gamma$ is a homomorphism.)

In general the representation will be by tuples of elements with an appropriate multiplication. (Following a suggestion of J. Neubüser, these can be realized by "monomial type" matrices.)

Let $\hat{\alpha}$ be the homomorphism defined on $U \gamma$ defined by $(u \gamma) \hat{\alpha}=u \alpha$ (this is well-defined since $\operatorname{ker} \gamma \leq$ $\operatorname{ker} \alpha$ ). It is surjective onto $C$, so there is a subgroup $\hat{V} \leq U \gamma$ which is the full preimage of $V \alpha$ under $\hat{\alpha}$. The quotient representation of $V$ as a subgroup of $G$ therefore is $(\gamma, \hat{V})$.

If $\alpha$ is a permutation action as well, and $V$ is a point stabilizer in this action, the resulting image $\hat{V}$ is a point stabilizer in the resulting permutation action $\gamma$ for the wreath product.

In practice, once we have computed a presentation of $U$, we will work with an isomorphic finitely presented group $\hat{U}$ and compute the further quotient $\alpha$ from the presentation of $\hat{U}$.

Thus, to evaluate $\alpha$ on elements of $U \leq G$ we must express these elements as words in the generators of $U$ which gave the presentation for $\hat{U}$. This can be achieved by the same Reidemeister rewriting process used to compute a presentation of $U$ in the first place.

Typically the rewritten presentation $\hat{U}$ will be unhandily large and one will apply Tietze transformations first to shrink it - see the survey [Havas and Robertson 1993]. To keep the connection to the original $U$ one therefore has to keep track of the Tietze transformations being done.

If the index of $U$ gets larger, a little bit of care has to be taken to avoid memory problems: By default the rewriting yields a word that also uses "secondary" generators (are defined as words on the "primary" generators). Expanding these as words using only the primary generators can result in an exponential growth of the word lengths. Instead, we compute the images of all secondary generators under $\alpha$ and rewrite immediately as an element of $U \alpha$. If elements of this image group are given in unique form (say as matrices or permutations) this exponential growth will not apply in this representation.

Finally, some care has to be taken if we compute quotient subgroups of $V<U<G$ when $V$ itself had been given by a quotient of $U$. The way we found the quotients it would be tempting to rewrite elements of $V$ as elements of $G$ via $U$. However this means that at an intermediate stage we would have to handle rewritten elements of $U$ as words, which (as mentioned above) can become extremely long. Instead it is much quicker to create a new augmented coset table for $V$ and to rewrite using this table.

\section{POLYCYCLIC-BY-FINITE GROUPS}

Definition 4.1. Let $\beta: G \rightarrow H$ be an epimorphism. A homomorphism $\delta: G \rightarrow N$.H with $\operatorname{ker} \delta \leq \operatorname{ker} \beta$ and $N \cong \operatorname{ker} \beta / \operatorname{ker} \delta$ is called a lift of $\beta$. We call $N$ the lift kernel of $\delta$.

Remark 4.2. Following the use in [Huppert 1967; Robinson 1996], we call an extension $1 \rightarrow N \rightarrow$ $N . H \rightarrow H \rightarrow 1$ an extension of $N$ by $H$. (This means that the image group of a lift of $\beta$ with lift kernel $N$ is an extension of $N$ by $G \beta$.)

We now consider the situation that $[G: U]$ is finite, $\beta$ is the permutation action of $G$ on the cosets of $U$ and that a homomorphism $\alpha: U \rightarrow U \alpha$ is obtained as a result of a polycyclic (or nilpotent) quotient algorithm applied to a presentation for $U$. (For finite groups, "polycyclic" is equivalent to "solvable".) Then $U \alpha$ is polycyclic and $\gamma: G \rightarrow U \alpha \succ G \beta$ is a lift of $\beta$ whose image is polycyclic-by-finite with the "finite" part given by $G \beta$.

Since polycyclic-by-finite groups are the largest class of groups for which an algorithmic theory is possible [Baumslag et al. 1991] a natural question which arises at this point is therefore whether this iterated quotient construction can help to expose all polycyclic-by-finite factor groups of $G$; respectively all such factors, where the top part is given by the image $G \beta$. 
Certainly all such lifts can be found by computing polycyclic quotients for $U=\operatorname{ker} \beta$, indeed the embedding theorem for wreath products is often given only for this situation (see [Huppert 1967, 15.9], for example).

On the other hand the number of generators and relators for a subgroup presentation grows with the subgroup index, and even reduction methods such as reduced Reidemeister-Schreier and Tietze transformations cannot remedy this fully. Therefore the index of $\operatorname{ker} \beta$ is often too big to make the computation of a presentation computationally feasible. The algorithmic question we face is thus: Given a homomorphism $\beta$, find a subgroup $U \geq \operatorname{ker} \beta$ of index as small as possible, such that a quotient algorithm applied to $U$ will find some (or all) finite by polycyclic factors that lift $G \beta$. This gives rise to:

Definition 4.3. Assume there is a lift $\delta: G \rightarrow H$ of $\beta$ whose lift kernel $N=(\operatorname{ker} \beta) \delta \triangleleft H$ is a polycyclic group. Take $U \leq G$ with $\operatorname{ker} \beta \leq U$. We say that $N$ is visible from $U$ if there is a homomorphism $\alpha$ on $U$ so that the image $U \alpha$ is polycyclic and that $\bigcap_{g \in G}(\operatorname{ker} \alpha)^{g} \leq \operatorname{ker} \delta$.

Certainly any polycyclic $N$ is visible from $\operatorname{ker} \beta$. We also observe that visibility does not solely depend on the image $U \beta$, but also on the type of the extension: Consider the case of $2 . A_{6}$ and $2 \times A_{6}$. In both cases there is a factor group $G \beta$ isomorphic $A_{6}$ with normal subgroup $N$ isomorphic $Z_{2}$. We consider a subgroup $U$ so that $U \beta=\operatorname{Stab}_{A_{6}}(1) \cong A_{5}$. In the first case, the image of $U$ in $H$ is the perfect group 2. $A_{5}$, so $N$ is invisible from $U$. In the second example, however the image of $U$ in $H$ is $2 \times A_{5}$, so there is a nontrivial solvable quotient $\alpha$ which makes $N$ visible.

For the general situation, let $\delta$ be a lift of $\beta$ and

$$
V:=U^{(\infty)} \delta=(U \delta)^{(\infty)}
$$

the smallest normal subgroup of $U \delta$ with solvable factor. Then visibility of $N$ means that $K:=V \cap N$ does not contain any nontrivial normal subgroup of $H$. We also set $M:=\langle V, N\rangle$. So $U \delta / M$ is the largest solvable quotient of $U \delta / N \cong U \beta$. Thus $M=X \delta$ where $X$ is the full preimage of $(U \beta)^{(\infty)}$ under $\beta$. Being an intersection of two normal subgroups, $K$ is normal in $U \delta$, so $U \delta / K$ is a subdirect product of
$U \delta / V$ with $U \delta / N$ with common factor $U \delta / M$. This is illustrated by the following diagram:

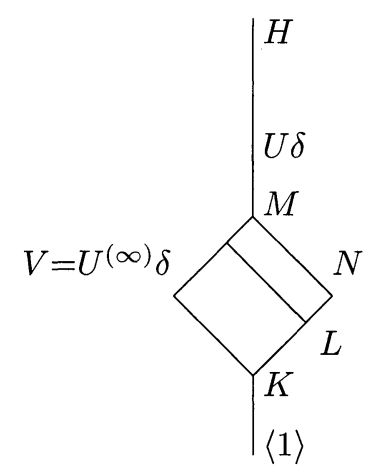

Now consider the easiest nontrivial case: Assume that $N$ is a minimal normal subgroup of $H$ and thus is elementary abelian. Then $N / K$ is abelian and thus $M^{\prime} \leq V$. Furthermore $N / K$ is central in $M / K$ and thus $V \triangleleft M$.

$N$ is a module for $G \beta \cong H / N$. When restricted (from being a $G \beta$-module) to $M \beta$, the module $N$ has a trivial factor. In other words: There is a subgroup $W \leq U$ (we can set for example $W:=M$ ) such that $U / W$ is solvable and that $W \beta$ is a subgroup of a vector stabilizer for the dual module of $N$. Let $L \leq N$ be an $M \beta$-submodule (considering $N$ as an $M \beta$-module by restriction) of codimension one with trivial factor module. Since $V \triangleleft M$, the factor $M / L$ has a complement (namely $\langle V, L\rangle / L$ ) to $N / L$.

Conversely, let $W \leq G$ be a subgroup such that $W \beta$ stabilizes a vector for the dual module of $N$. Then there must be a $W \beta$-submodule $L<N$ of codimension 1 , and thus $L \triangleleft W \delta$. In the factor $W \delta / L$, either the perfect residuum $(W \delta / L)^{(\infty)}$ intersects trivially with $N / L$ (and so $N$ is visible from $W$ and thus visible from any $U \triangleright W$ with $U / W$ solvable) or it contains $N / L$. In this second case, setting $P:=(W \delta) / L$, we have that $N / L \leq Z(P) \cap P^{\prime}$ and $P \cong(N / L) \cdot(W \beta)$. We call such an extension of a (nontrivial) $N / L$ by $W \beta$ Schurian and the induced epimorphism $\rho: W \rightarrow P$ a Schurian lift of $\left.\beta\right|_{W}$.

We have proved:

Lemma 4.4. If a known quotient $\beta$ of a finitely presented group $G$ lifts with the lift kernel isomorphic to a simple module $N$, and $W \leq G$ is a subgroup such that $W \beta$ is a vector stabilizer in the dual module of $N$, either $N$ is visible from every subgroup $U$ 
with $W \triangleleft U \leq G$ and $U / W$ solvable, or there is a Schurian lift of $\left.\beta\right|_{W}$.

Since we aim to "discover" $N$ (and thereby the new quotient $H$ ) we now develop a criterion for the existence of Schurian lifts:

\section{A. Relators in the Schur Cover}

To study this situation, we have to examine Schur Covers in more detail. Let $F$ be a free group, $G$ a group with projection $\gamma: F \rightarrow G$ and let $R=$ $\operatorname{ker} \gamma=\langle\mathcal{R}\rangle_{\mathrm{NT}}$. Assume that there is an epimorphism $\beta: G \rightarrow B$ onto a finite group $B$, denote the kernel of the projection of $F$ onto $B$ by $S=$ $\operatorname{ker}(\gamma \beta)$ and note that $R \leq S$. We finally assume that $\operatorname{ker} \beta \leq G^{\prime}$, that is the largest abelian quotient of $G$ is isomorphic to the largest abelian quotient of $B$.

We want to investigate, under which conditions there can be a Schurian lift $\mu: G \rightarrow Q$ of $\beta$ (that is we look for a quotient $Q$ of $G$ that is a Schurian extension of a normal subgroup by $G \beta$ ). For such a lift, we denote the corresponding epimorphisms by $\lambda: F \rightarrow Q$ and $\nu: Q \rightarrow B$.

Lemma 4.5. The quotient $F / R[S, F]$ is a Schurian extension of $S / R[S, F]$ by $B$ and is a quotient of $G$. For any Schurian lift $\mu$ of $\beta$ we have for the epimorphism $\lambda=\gamma \mu$ that $\operatorname{ker} \lambda \geq R[S, F]$.

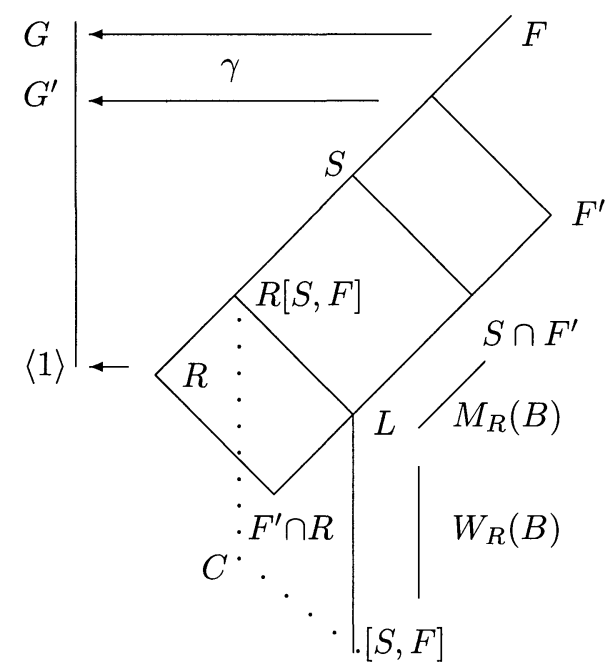

Proof. (See diagram for clarity.) Since $\operatorname{ker} \beta \leq G^{\prime}$, we have that $\left\langle R, F^{\prime}\right\rangle=\left\langle S, F^{\prime}\right\rangle$. This implies, setting $\lambda: F \rightarrow F / R[S, F]$, that $S \lambda \leq Z(F \lambda) \cap(F \lambda)^{\prime}$. Since $R \leq S$, the epimorphism $\lambda$ induces an epimorphism $\mu: G \rightarrow F \lambda$, and since $[S, F] \leq S$ this epimorphism is a Schurian lift of $\beta$.

On the other hand for any Schurian lift $\mu$ of $\beta$ (setting $\lambda=\gamma \mu$ ), $R \leq \operatorname{ker} \lambda$ is a condition for $\mu$ being a lift of $\beta$. Since $F \lambda$ is a Schurian extension of $S \lambda$ by $F \beta$ we have that $S \lambda \leq Z(F \lambda)$ and thus $[S, F] \leq \operatorname{ker} \lambda$.

Since every Schurian extension is a factor of a Darstellungsgruppe [Schur 1907, §2,III], we now consider Darstellungsgruppen and the multiplier

$$
M(B) \cong\left(S \cap F^{\prime}\right) /[S, F]
$$

of $G \beta$ :

Definition 4.6. Set $L:=R[S, F] \cap F^{\prime}$ and $W_{R}(B):=$ $L /[S, F]$. The factor

$$
\begin{aligned}
M_{R}(B):=\left(S \cap F^{\prime}\right) / L & \cong S / R[S, F] \\
& \cong M(B) / W_{R}(B)
\end{aligned}
$$

is called the $R$-fixed multiplier of $B$. (These definitions depend not only on $R$ and $B$, but also on the choice of $\beta$.)

This group $M_{R}(B)$ is the largest factor of the multiplicator $M(B)$ that can be realized compatibly to $\beta$ and $R$.

If $C$ is a complement to (the torsion subgroup) $L /[S, F]$ in $R[S, F] /[S, F]$, then $C$ also is a complement to $S \cap F^{\prime} /[S, F]$ in $S /[S, F]$. Thus $F / R[S, F]$ is a quotient of a Darstellungsgruppe $F / C$ of $B \cong$ $F / S$. In other words: We obtain the largest Schurian quotient by evaluating the relators $\mathcal{R}$ for $B$ in a suitable Darstellungsgruppe (namely $F / C$ ) of $B$. Here suitable means not only a suitable isomorphism type, but a suitable choice of the complement $C$ which influences the relator values in the Darstellungsgruppe.

Remark 4.7. If $B$ is a group which has several nonisomorphic Darstellungsgruppen and we take for $\mathcal{R}$ the relators of another Darstellungsgruppe not isomorphic to $F / C$, we see that $R[S, F]$ does not necessarily contain every complement $C$ to $S \cap F^{\prime}$. We therefore cannot compute $W_{R}(B)$ by evaluating the relator set $R$ in an arbitrary Darstellungsgruppe, the choice of a "suitable" group is crucial.

We get around this problem by working with $L$ instead which does not depend on the choice of $C$ and which together with $C$ will generate $R[S, F]$. 
Lemma 4.8. $\left\langle[S, F], R \cap F^{\prime}\right\rangle=R[S, F] \cap F^{\prime}=L$.

Proof. Since $[S, F] \leq S \cap F^{\prime}$ and $R \leq S$, both generating groups on the left hand side are in both groups on the right hand side of the equation. To see the converse, take $x \in R[S, F] \cap F^{\prime}$. Then there are $r \in R, s \in[S, F]$ and $f \in F^{\prime}$, such that $x=r s=f$. Since $[S, F] \leq F^{\prime}$, we see that $r=f / s \in F^{\prime}$ and thus $x=r \cdot s \in\left\langle R \cap F^{\prime},[S, F]\right\rangle$.

Since $\left[F:\left\langle R, F^{\prime}\right\rangle\right]=\left[G: G^{\prime}\right]<\infty$ and normal subgroup generators for $R$ and $F^{\prime}$ are known, Lemma 2.3 and Corollary 2.4 give a generating set $\mathcal{J}$ for $R \cap F^{\prime}$ as a normal subgroup.

Under an epimorphism from $F$ onto an (arbitrary) Darstellungsgruppe $D$, the subgroup $[S, F]$ maps to the trivial subgroup of $D$. Thus the set $\mathcal{J}$ will map to a normal subgroup generating set of (the image in $D$ ) of $\left\langle R \cap F^{\prime},[S, F]\right\rangle$ which is (the image in $D$ ) of $R[S, F]$. Furthermore, since the multiplier is central in $D$, the images of $\mathcal{J}$ are subgroup generators of this subgroup. We deduce:

Lemma 4.9. Let $D$ be an arbitrary Darstellungsgruppe for $G \beta, \nu: D \rightarrow G \beta$ the natural homomorphism and $\delta: F \rightarrow D$ be a projection of $F$ on $D$ such that $\gamma \beta=$ $\delta \nu$.

Let $\underline{\boldsymbol{d}}$ be the list of images of the free generators of $F$ under $\delta$. (If only $D$ and $\nu$ are given, one can get $\underline{\boldsymbol{d}}$ as preimages under $\nu$ of the images of the free generators of $F$ under $\gamma \beta$, this will define such a set $\delta$.)

Then $W_{R}(B) \cong\langle\mathcal{J} \delta\rangle \leq D$ (that is, it is the subgroup generated by the words in $\mathcal{J}$ evaluated in $\underline{\boldsymbol{d}}$ ).

Proof. Since $\gamma \beta=\delta \nu, \operatorname{ker} \delta \leq S$ and so $\operatorname{ker} \delta$ can be considered to be in place of the complement subgroup $C$ considered above. Since $W_{R}(B) \leq M(B)$ is central in $D$, normal subgroup generators suffice.

To express J explicitly, we have to apply Lemma 2.3 for the homomorphisms $\varphi:=\gamma: F \rightarrow F / R \cong G$ and $\psi: F \rightarrow F / F^{\prime}$. Since $F / F^{\prime}$ is abelian, the set $Y_{2}$ - sufficiently many conjugates of relators $r^{f}$ $(r \in \mathcal{R}, f \in F)$ (remember that $R=\langle\mathcal{R}\rangle_{\mathrm{NT}}$ ), such that the images of these conjugates in $F / F^{\prime}$ will generate $(\operatorname{ker} \varphi) \psi$ as a subgroup - consists only of relators and no proper conjugates are needed. Also the relations of type (2-3) (the conjugation action of $F / F^{\prime}$ on $\left.\left\langle Y_{2}\right\rangle \triangleleft F / F^{\prime}\right)$ become trivial. Thus we get $\mathcal{J}=\left\{r\left(Y_{2}\right) \mid r \in \mathcal{R}_{F}\right\}$ (where $\mathcal{R}_{F}$ are relators for $(\operatorname{ker} \varphi) \psi \triangleleft F / R$ in the generating set $Y_{2} \psi$ ).

Since $\left[F: R \cdot F^{\prime}\right]$ is finite, the subgroup $R F^{\prime} / F^{\prime} \leq$ $F / F^{\prime}$ is torsion free abelian and the relations in $\mathcal{R}_{F}$ are just commutators and "integral linear dependencies". We denote these linear dependencies among the images of the $Y_{2}$ by $\mathcal{R}_{\text {lin }}$. When representing the images of $Y_{2}$ in $F / F^{\prime}$ by coefficient vectors these dependencies can be deduced from a Smith normal form of the matrix formed by these vectors.

Since commutator relations hold automatically in $M(B) \leq D$, we obtain $\mathcal{J} \delta$ (and thus $W_{R}(B)$ ) by evaluating the relators $\mathcal{R}_{\text {lin }}$ in the images $Y_{2} \delta$. We obtain these images $Y_{2} \delta$ by evaluating the relators in $\mathcal{R}$ (the relators for $G$ ) in the free generator images $\underline{\boldsymbol{d}} \subset D$.

In other words:

Theorem 4.10. If $D$ is any Darstellungsgruppe of $B$ with $D / K \cong B$ and $\left[G: G^{\prime}\right]=\left[B: B^{\prime}\right]$, the lift kernel of a largest Schurian lift of $\beta$ that is a homomorphism of $G \cong F / R$ is given by $M_{R}(B) \cong K / W_{R}(B)$, where $W_{R}(B)=\left\langle r\left(Y_{2}\right) \mid r \in \mathcal{R}_{\operatorname{lin}}\right\rangle$ and $Y_{2}=\{r(\underline{\boldsymbol{d}}) \mid$ $r \in \mathcal{R}\}$.

\section{B. Computing $W_{R}(B)$ and $M_{R}(B)$}

In practice we want to compute $W_{R}(B)$ and the factor $M_{R}(B)$ for a given $B, G$ and $\beta: G \rightarrow B$ without having to construct a Darstellungsgruppe $D$ first.

We note first that we can consider the different $p$-parts of $M(B)$ and of $W_{R}(B)$ separately.

For a given $B$ and $p$, the algorithm in [Holt 1984] will compute a PC presentation for a lift $L$ of a $p$ Sylow subgroup $P$ of $B$ with $M_{p}(B)$, together with an epimorphism $\pi$ of this lift onto $P$. (This lift $L$ however is not necessarily isomorphic to a Sylow subgroup of a Darstellungsgruppe of $B$.)

This lift corresponds to a cocycle $\varphi \in H^{2}(P, \mathbb{C})$. Furthermore [Holt 1985] shows that the corestriction $\psi:=\operatorname{cor}_{P, B}(\varphi) \in H^{2}(B, \mathbb{C})$ corresponds to a $p$-Darstellungsgruppe $\hat{B}$ of $B$ :

If $\hat{b}$ denotes the choice of a fixed representative in $\hat{B}$ for $b \in B$, we conclude that $\hat{b}_{i} \hat{b}_{j}=\widehat{\left(b_{i} b_{j}\right)} \psi\left(b_{i}, b_{j}\right)$ for $b_{i}, b_{j} \in B$.

By iteration, we can therefore use the algorithm for evaluating $\psi$ via the a "transfer"-like sum given in [Holt 1985] to obtain for each relator $r \in \mathcal{R}$ of $G$ a value $m_{r}$ in the $p$-part of $M(B)$. 
To compute the relations $\mathcal{R}_{\text {lin }}$, we collect (abelianized) coefficient vectors for all the relators $r$ in a matrix $A$ and compute the Smith normal form $S$ of $A$. The transformation matrix $P$ (when $A=P S Q$ ) then gives the linear relations which yield the $p$-part of $W_{R}(B)$.

We then get $M_{R}(B)$ as the factor $M(B) / W_{R}(B)$.

\section{C. Application to the Problem of Finding Lifts}

We now return to the problem of finding all factor groups of $G$ which are lifts of $G \beta$ with a simple module:

Let $A$ be the simple module of $G \beta$ for which we want to find lifts. Let $U \leq G$ such that $U \beta$ is a vector stabilizer for a nontrivial vector in the dual of $A$ and let $U \triangleleft \tilde{U} \leq G$ with $\tilde{U} / U$ a solvable supergroup of $U$.

By rewriting (possibly in several steps via intermediate subgroups between $\tilde{U}$ and $G$, which we can again find via their images under $\beta$ ) we compute a presentation of $\tilde{U}$. Let $H:=\langle X \mid \mathcal{R}\rangle$ be the corresponding finitely presented group. The rewriting process also gives an isomorphism $\zeta: \tilde{U} \rightarrow H$, which permits us to evaluate $\left.\beta\right|_{\tilde{U}}$ on the isomorphic group $H$. From the subgroup presentation we compute the largest solvable quotient $\alpha$ of $\tilde{U}$ so that $U / \operatorname{ker} \alpha$ is elementary abelian.

If $\operatorname{ker} \alpha \nsupseteq \operatorname{ker} \beta$, the induced representation $\gamma:=$ $\alpha \uparrow_{(\beta)}^{G}$ exposes a proper lift $Q$ of $\beta$ with a normal subgroup with a factor isomorphic $A$, which is a quotient of $G$.

Furthermore, from the presentation we determine $M_{R}(U \beta)$. If this group is not trivial, and not all of it can be realized as a quotient of $Q$, there is a Schurian lift of $\tilde{U} \beta$ that is a quotient of $H$. This lift might correspond to another factor group of $\operatorname{ker} \beta$ which is isomorphic to $A$ (and thus another lift of $\beta$ with lift kernel isomorphic to $A$ ).

In this case we have to find a new subgroup $U_{2}$ of $U$, for which the lift will be not Schurian any longer. In many cases (for example for the obvious choice of a non- $p$ Sylow subgroup) however the index $\left[G: U_{2}\right]$ will be prohibitively large. Further work will be needed to make the construction efficient and to represent the corresponding lift.

Remark 4.11. It is worth mentioning that we can be lucky and a lift obtained by inducing a representa- tion of $U$ to $G$ will simultaneously expose the largest possible Schurian lift (and so no search for a $U_{2}$ is necessary). We will see an example of this in Section $5 \mathrm{C}$.

Remark 4.12. In practice frequently not just a single module but a sequence of modules is considered. Thus we might find several different lifts. In this case we can in each step replace $Q$ by the subdirect product of $Q$ and the largest lift known so far.

It is possible, that several of those lifts have the same kernel. In this case (which can be checked for by computing the quotients of the image of one subgroup in the lift of the other) it is sufficient to construct only one lift.

Finally, in the case that lifts for several modules are to be considered, it can happen that a vector stabilizer for the one module is contained in a vector stabilizer for the other module. In this case it is worth noting that if $U \leq V \leq G$ everything visible from $V$ or every Schurian lift recognizable from $M_{R}(V)$ will be visible, respectively recognizable, from $U$ as well. So only the smaller vector stabilizer need to be considered.

\section{AN EXAMPLE}

D. Pasechnik [1998] has posed a question about the group

$$
\begin{aligned}
G=\langle a, b, c, & d, e, f, g, h, i, j \mid \\
& a^{2}, b^{2}, c^{2}, d^{2}, e^{2}, f^{2}, g^{2}, h^{2}, i^{2} \\
& (a b)^{4},(a c)^{5},(a d)^{3},(a e)^{2},(a f)^{2} h \\
& (a g)^{2} i,(a h)^{2},(a i)^{2},(b c)^{2} d g,(b d)^{2}, \\
& (b e)^{2},(b f)^{2} d,(b g)^{2},(b h)^{3},(b i)^{2} e,(c d)^{2}, \\
& (c e)^{2} d,(c f)^{2},(c g)^{2},(c h)^{2} g,(c i)^{2} f g \\
& (d e)^{2},(d f)^{2},(d g)^{2},(d h)^{2} f,(d i)^{2} g \\
& (e f)^{2} g,(e g)^{2},(e h)^{2} i,(e i)^{2},(f g)^{2} \\
& (f h)^{2},(f i)^{2},(g h)^{2},(g i)^{2},(h i)^{2}, j^{2}, \\
& \left.(d j)^{2} e,[j, a d],(c j)^{3},(j f)^{2} g i,(j b)^{2} e g\right\rangle,
\end{aligned}
$$

[Baumeister et al. 2000], which has a quotient isomorphic $M c L$ exposed by the extra relators $(a c b)^{8}$ and $(j c a)^{7}$. Let $N$ be the kernel of this quotient $\beta: G \rightarrow M c L$. The question mentions a known lift kernel $3^{23}$ and asks whether this is the largest 3-lift kernel. 
Here we would like to find possible lifts with a GF(3)-module.

The modular ATLAS [Jansen et al. 1995] lists the degrees of the smallest dimensional irreducible $\mathrm{GF}(3)$-modules of $M c L$ as 1, 21, 104, 104 (the dual) and 210 .

(The reason we stop here and do not consider the other representations is purely practical: The involved calculations turn out to be already at the limit of what was possible with the computers available to the author. By the techniques of this article it would also be possible to consider the remaining modules for $M c L$. It is to be expected, however, that such calculations would become even harder, while not illustrating additional features of the method.)

Explicit matrices for these representations can be found in [Wilson et al. 1996].

By investigating the behaviour of these representations for some large subgroups of $M c L$ (all calculations were done in GAP [GAP 2000]), we find subgroups which stabilize vectors in the dual modules:

\begin{tabular}{lllr} 
& Subgroup & Specification & Dimensions \\
\hline 0 & $M c L$ & & 1 \\
1 & $U_{4}(3)$ & point stabilizer & 21 \\
2 & $3^{4} \cdot A_{6}$ & 2-point stabilizer & $104 \mathrm{a}, 210$ \\
3 & $3^{1+4}: 2 . S_{4}$ & pt. stab. in $N(3 A)$ & $104 \mathrm{~b}$
\end{tabular}

\section{A. A Proper Lift}

The group $G$ itself is perfect, so all trivial modules must come from Schurian lifts. Since $M c L$ is large, and we will examine proper subgroups, by Remark 4.12 it is not worth to compute $M_{R}(M c L)$ now; we will get the same information from subgroups.

Let $U$ be the preimage of $U_{4}(3)$ under $\beta$. Using Reidemeister-Schreier rewriting and Tietze transformations we find a presentation for $U$ on 4 generators with 49 relators. Abelianizing this presentation shows that $U$ is perfect as well. We also know, that $M\left(U_{4}(3)\right) \cong 3^{2} \times 4$. A calculation now shows that the 3-part of $W_{R}\left(U_{4}(3)\right)$ is trivial and thus the 3 -part of $M_{R}\left(U_{4}(3)\right)$ is $3^{2}$. We therefore know, that there will be Schurian lifts which show that $\operatorname{ker} \beta$ must have factor groups that are $M c L$-modules, for whose duals $U_{4}(3)$ is a vector stabilizer (for example the trivial or the 21-dimensional module).

We next look at the subgroup of type $3^{4}$. $A_{6}$, which is a point stabilizer in $U_{4}(3)$ in the action on 275 points for one orbit of length 112 . Let $V$ be the full preimage in $G$ of this point stabilizer. By the same methods we find a presentation for $V$ on 10 generators with 925 relators (of total length $\sim 120000$ ).

Representing the wreath product of the action of $U$ on the cosets $V \backslash U$ and of $G$ on $U \backslash G$ as a permutation group we obtain a quotient representation of $V$ by a permutation group of degree 30800 .

This group $V$ has a cyclic quotient of order 3 . (Indeed, $V^{\prime}$ is perfect of index 3 in $V$, so this is the largest solvable quotient of $V$.) The wreath product of this action $\alpha$ with the action $\rho$ on the cosets of $V$ yields a lift $\varphi=\alpha \uparrow_{(\rho)}^{G}$ with an image group of order $3^{104} \cdot|M c L|$. (This disproves the original conjecture about a maximal 23-dimensional lift kernel.) The lift kernel $3^{104}$ is an irreducible module for $M c L$.

Calculating permutation generators for this group $3^{104} \cdot|M c L|$ took about 1 hour on an UltraSPARC 10 when starting with the presentation for $G$. Computation of a stabilizer chain (and thus determining the order and the dimension of the module) then took 3 hours (and required about $500 \mathrm{MB}$ of storage).

Remark 5.1. In the resulting permutation representation on 92400 point, the normal subgroup $3^{104}$ becomes a subgroup of $3^{30800}$ acting intransitively with orbits of length 3 . Therefore the resulting permutation group will have base length at least 104. This, and the large degree of the permutation representation indicate, that the representation as a permutation group is not optimal for such quotients and indeed a representation as generic wreath product might be more suitable.

The multiplier of $3^{4}$. $A_{6}$ has structure $2 \times 3^{3}$. Again, a calculation shows that the 3 -part of $W_{R}\left(3^{4} \cdot A_{6}\right)$ is trivial (and thus the 3-part of $M_{R}\left(3^{4} \cdot A_{6}\right)$ is $3^{3}$ ). This shows that this is not the maximal lift of $\beta$ with abelian lift kernel.

\section{B. A Second Lift}

Next, we look at a subgroup of type $3^{1+4}: 2 . S_{4}$. (We find this as point stabilizer in a subgroup of type $3^{1+4}: 2 . S 5$, which in turn is the normalizer of a cyclic 
subgroup of order 3 in $M c L$.) Denote its preimage in $G$ by $X$.

We find a presentation for $X$ on 9 generators with 1223 relators (of total length $\sim 240000$ ).

The abelian quotient $X / X^{\prime}$ has order 6 . However, if we map $X$ in the already mentioned quotient $3^{104} . M c L$, we get an image $X \varphi$ with $\left[X \varphi: X \varphi^{\prime}\right]=2$. So the quotient of $X$ of order 3 will yield another quotient of $G$ which exposes another abelian quotient part of $N$.

Here we get a permutation representation of degree 231000 . The resulting lift $\psi$ of $\beta$ has an image group of order $3^{127} \cdot|M c L|$. The lift kernel $3^{127}$ is an uniserial $M c L$-module with structure $3^{104} \cdot 3.3^{21} .3$.

Remark 5.2. Permutations on 231000 points take up almost $1 \mathrm{MB}$ each. Since the resulting group has base length 127, a strong generating set therefore will take at least $127 \mathrm{MB}$ of memory, a stabilizer chain will require even more. In fact it turns out that the default stabilizer chain routine in GAP will run out of memory (the author was not willing to compile a "static" binary to permit the use of more than $512 \mathrm{MB}$ of memory). Instead, intermediate results were represented as words in the generators. The flexible type system of GAP [Breuer and Linton 1998] made it possible to have those new wordelements to look to the system like permutations, so that the existing code for stabilizer chain computation could still be used.

We note that the module - being uniserial - has no factor isomorphic $3^{104}$. The quotient $3^{104}$ found before therefore must be a different quotient of $N$. (In fact MeatAxe methods [Holt et al. 1995] show that both 104-dimensional modules are dual to each other.)

Thus we know that $N$ must have an elementary abelian 3-quotient of size at least $3^{231}$.

The same technique as above was used to construct generators for the module $3^{127}$ which was required to compute the composition structure.

The multiplier of $3^{1+4}: 2 . S_{4}$ is of type $3 \times 3$ and again a calculation of $W_{R}\left(3^{1+4}: 2 . S_{4}\right)$ shows, that the whole of this is realizable via lifts of $\beta$.

Nonetheless, an explicit calculation in the image group $G \psi$ shows that the whole of $M_{R}(X \beta)$ is realizable in this quotient. (This is the example promised in Remark 4.11.)
It is also possible to combine both quotients into a subdirect product as an intransitive group of degree 323400 . Verifying that this product has size $3^{231} \cdot|M c L|$, however, stretches available computing resources, requiring about $550 \mathrm{MB}$ of workspace.

Remark 5.3. Since $X \beta \cong 3^{1+4}: 2 . S_{4}$ is solvable one could try to use a solvable quotient algorithm now to find the largest quotient of $X$ whose restriction to $N$ is elementary abelian. However, we already know that the this factor is at least $3^{231}$, which would make such a calculation very hard.

Moreover, the approach of [Plesken 1987; Brückner 1998] is unsuitable in this situation, since the composition factors of the large module restricted to $3^{1+4}: 2 . S_{4}$ are of dimension at most 4 . Building already a 231 dimensional module from such small parts becomes unfeasibly hard. Indeed the GAP implementation based on [Plesken 1987] managed to construct a quotient of size $11664 \cdot 3^{37}$ over a day and then became that slow that the calculation was stopped.

The approach of [Niemeyer 1994] ought to work better in this situation, however (in part since the author had severe problems to compile a working version of all required programs) this has not yet been tried.

\section{C. Exposal of Schurian Lifts in other Quotients}

To find out if the lifts found also expose Schurian lifts, we map the subgroups $G, U, V$ and $X$ under $\varphi$ and $\psi$ and compute the commutator subgroups with the corresponding lift kernels. (That is for each subgroup $A \leq G$ we compute the commutator $[A \lambda, N \lambda] \leq N \lambda$.) We get the following table, where $q:=[N \lambda:[A \lambda, N \lambda]]$ and $r:=\left[A \lambda:(A \lambda)^{\prime}\right]$ :

\begin{tabular}{clcccc} 
& $A \beta$ & $M_{R}(A \beta)$ & Lift $\lambda$ & $q$ & $r$ \\
\hline$G$ & $M c L$ & 3 & $\psi$ & 3 & 1 \\
$U$ & $U_{4}(3)$ & $3^{2}$ & $\psi$ & $3^{2}$ & 1 \\
$V$ & $3^{4} \cdot A_{6}$ & $3^{3}$ & $\psi$ & $3^{3}$ & 1 \\
$X$ & $3^{1+4}: 2 . S_{4}$ & $3^{2}$ & $\psi$ & $3^{3}$ & 6 \\
$X$ & $3^{1+4}: 2 . S_{4}$ & $3^{2}$ & $\varphi$ & 1 & 2
\end{tabular}

These results show that all Schurian lifts for the subgroups $U \beta$ and $V \beta$ (and so also all Schurian lifts for $G \beta$ ) are exposed as parts of $\psi$. (We implicitly also deduced that the 3-part of $M_{R}(M c L)$ has size 3.) Since $M_{R}\left(3^{1+4}: 2 . S_{4}\right)$ is already realized in full 
in the image under $\psi$, there cannot be any extra Schurian lift for the module 104b.

We therefore discovered all lifts of $\beta$ whose lift kernel has a quotient isomorphic to a module of dimension up to 210 .

\section{ACKNOWLEDGEMENTS}

I would like to thank Derek Holt, Joachim Neubüser and Werner Nickel for helpful discussions. I'm also grateful to Steve Linton and Dave Alden for making larger computers available to me, which were used for the computations with the high-degree permutation groups.

Finally I'm indebted to the referee's careful reading and helpful comments.

\section{REFERENCES}

[Baumeister et al. 2000] B. Baumeister, A. A. Ivanov, and D. V. Pasechnik, "A characterization of the Petersen-type geometry of the McLaughlin group", Math. Proc. Cambridge Philos. Soc. 128:1 (2000), 2144.

[Baumslag et al. 1991] G. Baumslag, F. B. Cannonito, D. J. Robinson, and D. Segal, "The algorithmic theory of polycyclic-by-finite groups", J. Algebra 142:1 (1991), 118-149.

[Breuer and Linton 1998] T. Breuer and S. Linton, "The GAP 4 type system: Organising algebraic algorithms", pp. 38-45 in Proceedings of the 1998 International Symposium on Symbolic and Algebraic Computation (ISSAC '98) (Rostock, Germany, 1998), edited by O. Gloor, ACM Press, New York, 1998.

[Brückner 1998] H. Brückner, Algorithmen für endliche auflösbare Gruppen und Anwendungen, Dissertation, Rheinisch-Westfälische Techn. Hochschule, Aachen, Germany, 1998.

[Celler and Neunhöffer 1999] F. Celler and M. Neunhöffer, "XGAP: GAP4 share package", software, 1999. See http://www-gap.dcs.st-and.ac.uk/gap/Share/ xgap.html.

[GAP 2000] The GAP group, GAP: Groups, algorithms, and programming, Version 4.2, RWTH Aachen and University of St. Andrews, 2000. See http:// www-gap.dcs.st-and.ac.uk/ gap.

[Havas 1974] G. Havas, A Reidemeister-Schreier program (Canberra, 1973), edited by M. F. Newman, Lecture Notes in Math. 372, Springer, Berlin, 1974.
[Havas and Robertson 1993] G. Havas and E. F. Robertson, "Application of computational tools for finitely presented groups", pp. 29-39 in Groups and computation (New Brunswick, NJ, 1991), edited by L. Finkelstein and W. M. Kantor, DIMACS Ser. Disc. Math. Theoret. Comput. Sci. 11, Amer. Math. Soc., Providence, RI, 1993.

[Holt 1984] D. F. Holt, "The calculation of the Schur multiplier of a permutation group", pp. 307-319 in Computational group theory (Durham, 1982), edited by M. D. Atkinson, Academic Press, 1984.

[Holt 1985] D. F. Holt, "A computer program for the calculation of a covering group of a finite group", $J$. Pure Appl. Algebra 35:3 (1985), 287-295.

[Holt and Rees 1992] D. F. Holt and S. Rees, "Testing for isomorphism between finitely presented groups", pp. 459-475 in Groups, combinatorics and geometry, edited by M. W. Liebeck and J. Saxl, LMS Lecture Note Series 165, Cambridge Univ. Press, Cambridge, 1992.

[Holt and Rees 1993] D. F. Holt and S. Rees, "A graphics system for displaying finite quotients of finitely presented groups", pp. 113-126 in Groups and computation (New Brunswick, NJ, 1991), edited by L. Finkelstein and W. M. Kantor, DIMACS Ser. Disc. Math. Theoret. Comput. Sci. 11, Amer. Math. Soc., Providence, RI, 1993.

[Holt et al. 1995] D. F. Holt, C. R. Leedham-Green, E. A. O'Brien, , and S. Rees, "Smash: matrix groups and $G$-modules", GAP software package, 1995.

[Hulpke 1996] A. Hulpke, Konstruktion transitiver Permutationsgruppen, Ph.d. thesis, Rheinisch-Westfälische Technische Hochschule, Aachen, Germany, 1996.

[Huppert 1967] B. Huppert, Endliche Gruppen, I, Grundlehren der Math. Wissenschaften 134, Springer, Berlin, 1967.

[Jansen et al. 1995] C. Jansen, K. Lux, R. Parker, and R. Wilson, An atlas of Brauer characters, Oxford University Press, New York, 1995. Oxford Science Publications.

[Krasner and Kaloujnine 1951] M. Krasner and L. A. Kaloujnine, "Produit complet des groupes de permutations et problème d'extension de groupes II", Acta Sci. Math. (Szeged) 14 (1951), 39-66.

[Leedham-Green et al. 1991] C. R. Leedham-Green, C. E. Praeger, and L. H. Soicher, "Computing with group homomorphisms", J. Symbolic Comput. 12 (1991), 527-532. 
[Lo 1998] E. H. Lo, "A polycyclic quotient algorithm", J. Symbolic Comput. 25:1 (1998), 61-97.

[Macdonald 1974] I. D. Macdonald, "A computer application to finite p-groups", J. Austral. Math. Soc. Ser. A 17 (1974), 102-112.

[Magnus 1997] The New York Group Theory Cooperative, "Magnus: A system for exploring infinite groups", software, 1997. See http://zebra.sci.ccny.cuny.edu/web/.

[Magnus et al. 1966] W. Magnus, A. Karrass, and D. Solitar, Combinatorial group theory: Presentations of groups in terms of generators and relations, Interscience Publishers, New York, 1966.

[Neubüser 1982] J. Neubüser, "An elementary introduction to coset table methods in computational group theory", pp. 1-45 in Groups - St. Andrews 1981, edited by C. M. Campbell and E. F. Robertson, LMS Lecture Note Series 71, Cambridge Univ. Press, Cambridge, 1982.

[Nickel 1996] W. Nickel, "Computing nilpotent quotients of finitely presented groups", pp. 175-191 in Geometric and computational perspectives on infinite groups (Minneapolis and New Brunswick, 1994), edited by G. Baumslag et al., DIMACS Ser. Disc. Math. Theoret. Comput. Sci. 25, Amer. Math. Soc., Providence, 1996.

[Niemeyer 1994] A. C. Niemeyer, "A finite soluble quotient algorithm", J. Symbolic Comput. 18:6 (1994), 541-561.

[Pasechnik 1998] D. Pasechnik, "Abelian factors of the kernel of a homomorphism", 1998. See http://
www-gap.dcs.st-and.ac.uk/gap/Forum/Pasechni.1/ Dmitrii.1/Re__abel.1/1.html (note double underline).

[Plesken 1987] W. Plesken, "Towards a soluble quotient algorithm", J. Symbolic Comput. 4:1 (1987), 111-122.

[Remak 1930] R. Remak, "Über die Darstellung der endlichen Gruppen als Untergruppen direkter Produkte", J. Reine Angew. Math. 163 (1930), 1-44.

[Robinson 1996] D. J. S. Robinson, A course in the theory of groups, 2nd ed., Springer, New York, 1996.

[Schur 1907] I. Schur, "Untersuchungen über die Darstellung der endlichen Gruppen durch gebrochene lineare Substitutionen", J. Reine Angew. Math. 132 (1907), 85-137.

[Sims 1994] C. C. Sims, Computation with finitely presented groups, Cambridge Univ. Press, New York, 1994

[Todd and Coxeter 1936] J. A. Todd and H. S. M. Coxeter, "A practical method for enumerating cosets of a finite abstract group", Proc. Edinburgh Math. Soc. 5 (1936), 26-34.

[Wamsley 1974] J. W. Wamsley, "Computation in nilpotent groups (theory)", pp. 691-700 in Proceedings of the Second International Conference on the Theory of Groups (Canberra, 1973), edited by M. F. Newman, Lecture Notes in Math. 372, Springer, Berlin, 1974.

[Wilson et al. 1996] R. A. Wilson, P. Walsh, J. Tripp, I. Suleiman, S. Rogers, R. Parker, S. Norton, S. Linton, and J. Bray, "Atlas of finite group representations", online database, University of Birmingham, UK, 1996. See http://for.mat.bham.ac.uk/atlas/.

Alexander Hulpke, Department of Mathematics, The Ohio State University, 231 W. 18th Ave., Columbus, OH 43210, United States (ahulpke@math.ohio-state.edu). Current address: Department of Mathematics, Colorado State University, Fort Collins, CO 80523, United States (hulpke@math.colostate.edu)

Received October 27, 2000; accepted in revised form January 24, 2001 
